\title{
A validated stigma scale measures decreased HIV-related stigma among men in a community- based HIV prevention services program in rural Maharashtra, India
}

Ashok Dyalchand

From $16^{\text {th }}$ International Symposium on HIV and Emerging Infectious Diseases

Marseille, France. 24-26 March 2010

\section{Background}

To validate an HIV stigma scale among men in a high HIV-prevalent rural Indian district, and measure sixmonth changes in stigma after introducing an HIV prevention services program.

\section{Methods}

Between August 2006 and April 2007, a community HIV-related behavioural change and HIV testing services program was initiated in rural Aurangabad district, Maharashtra, to increase HIV services knowledge, reduce HIV stigma, and increase testing utilization. A questionnaire was administered to random cross-sectional samples of 400 adult men age 18-49 at Baseline and in the Post-Intervention and a separate post-Control community. Principle component factor analysis was used to develop a 14-item stigma scale. Stigma indices were calculated and dichotomized.

\section{Results}

Factor analysis consistently identified 3 HIV stigma subscales in each sample population. From Baseline to Post-Intervention, high stigma levels significantly decreased Overall ( $42 \%$ vs. $20 \%$ ), for Fear of HIV Transmission (33\% vs 14\%), and for Perception of Enacted Stigma $(34 \%$ to $14 \%)(\mathrm{p}<0.001)$. High stigma levels increased for Moral Judgements (79\% vs $86 \%, \mathrm{p}<0.05$ ). High stigma correlated independently with low education (AOR 2.7, 95\% CI 1.6-4.3), and low HIV knowledge (AOR 3.5, 95\%CI 2.2-5.5). Odds of high stigma reduced

Correspondence: dyalchand@gmail.com

Institute of Health Management, Pachod, Pune, India over $45 \%$ with participation in the Intervention program (AOR 0.54, 95\%CI (0.36-0.82)).

\section{Discussion}

This HIV stigma scale, identifying three stigma subscales consistent with other studies, was validated in $3 \mathrm{com}$ munity-based samples of rural men in. The HIV prevention services program reduced high HIV stigma overall and in HIV knowledge-related stigma domains. The program's current efforts include addressing Moral Judgment attitudes and longer-term study for impact on HIV testing utilization. Measurably reducing HIVrelated stigma is essential for HIV prevention in rural.

This HIV stigma scale, identifying three stigma subscales consistent with other studies, was validated in 3 community-based samples of rural men in India. The HIV prevention services program reduced high HIV stigma overall and in HIV knowledge-related stigma domains. The program's current efforts include addressing Moral Judgment attitudes and longer-term study for impact on HIV testing utilization. Measurably reducing HIV-related stigma is essential for HIV prevention in rural India.

Published: 11 May 2010

doi:10.1186/1742-4690-7-S1-P107

Cite this article as: Dyalchand: A validated stigma scale measures decreased HIV-related stigma among men in a community-based HIV prevention services program in rural Maharashtra, India. Retrovirology 2010 7(Suppl 1):P107. 